\title{
Differentially coherent code acquisition in the MIMO-aided multi-carrier DS-CDMA downlink
}

\author{
S.H. Won and L. Hanzo
}

\begin{abstract}
Both differentially coherent and non-coherent code acquisition schemes designed for the multiple-input multiple-output (MIMO)-aided multi-carrier (MC)-DS-CDMA downlink are analysed, when communicating over uncorrelated Rayleigh channels. The attainable mean acquisition time (MAT) performance is studied as a function of both the number of multiple transmit/multiple receive antennas and that of the number of subcarriers. It is demonstrated that in contrast to the expectations, when the number of multiple transmit antennas and/or that of the subcarriers is increased in both the differentially coherent and the non-coherent code acquisition scenarios, the achievable MAT deteriorates over the entire signal-to-interference plus noise ratio (SINR) per chip $\left(E_{c} / I_{o}\right)$ range considered, except for the scenario of single-carrier (SC)-DS-CDMA using $P=2$ transmit antennas and $R=1$ receive antenna. As expected, the degree of performance degradation depends upon the specific scheme and the $E_{c} / I_{o}$ ratio considered, although paradoxically, the correctly synchronised MIMO-aided system is capable of attaining its target bit error ratio performance at reduced SINR values.
\end{abstract}

\section{Introduction}

Multiple Transmit/Multiple Receive (MTMR) antennas adopted in the downlink of wireless systems constitutes a powerful technique of reducing the detrimental effects of fading by achieving a substantial diversity gain. Alternatively, MTMR antennas are capable of increasing the achievable throughput with the aid of BLAST-type spatial multiplexing schemes, when communicating over time-variant multi-path fading environments [1]. Furthermore, employing multiple subcarriers in the downlink of wireless systems exhibits significant benefits in terms of an increased flexibility, as argued in [2-4]. In recent years, diverse combinations of single-carrier code division multiple access (SC-CDMA) and orthogonal frequency division multiplexing (OFDM) [5] have attracted research efforts [2-5]. In inter-cell synchronous CDMA systems, the mobile station's (MS) receiver must be capable of synchronising the timing of a locally generated pseudonoise (PN) spreading sequence with that of the desired user's PN sequence contaminated by the interfering multi-user signals. Hence considerable research efforts have been devoted to the design of code acquisition techniques contrived for single-input single-output (SISO) systems [4, $6,7]$ in terms of the optimisation of the mean acquisition time (MAT). The code acquisition performance of multicarrier (MC) DS-CDMA attained with the aid of serial search-based schemes has been investigated in [4, 8]. In order to characterise the beneficial effects of multiple transmit antennas in terms of both the achievable correct detection and false alarm probability, the results of $[9,10]$ outlined the characteristics of a serial search-based code

(C) The Institution of Engineering and Technology 2007

doi:10.1049/iet-com:20060161

Paper first received 28th March and in revised form 21st November 2006

The authors are with the School of ECS, University of Southampton, Southampton SO17 1BJ, UK

E-mail: 1h@ecs.soton.ac.uk acquisition scheme in the context of the multiple transmit antenna aided SC-DS-CDMA downlink. However, there is a paucity of in-depth studies on the fundamental characteristics of code acquisition schemes designed for an MTMR antenna assisted MC-DS-CDMA system in the context of differentially coherent code acquisition schemes [9, 10]. Hence, solving this open problem is the main objective of the present paper. Similar to non-coherent code acquisition schemes $[4,6]$, no prior information concerning the absolute carrier phase is necessary for the operation of differentially coherent code acquisition schemes $[11,12]$. An additional benefit of using a differentially coherent code acquisition scheme is that it is capable of attaining a better performance than its non-coherent counterpart $[4,6,11,12]$. Here we adopted the full-period correlation (FPC) based scheme of $[11,12]$ in an effort to analyse the characteristics of serial search-based differentially coherent code acquisition in the MTMR antenna assisted MC-DS-CDMA downlink. Furthermore, in the verification mode of the differentially coherent scheme described in [13], the characteristics of the MIMO-assisted code acquisition schemes employing a double dwell serial search (DDSS) technique were analysed. Against this backdrop, in this treatise, we examine both serial search-based differentially coherent and noncoherent code acquisition schemes designed for MTMR antenna assisted MC-DS-CDMA systems. More explicitly, we quantify both the correct detection probability as well as the false alarm probability as a function of both the SINR per chip and that of the number of MTMR antennas as well as that of the number of subcarriers. Furthermore, we characterise the MAT against the SINR per chip performance parameterised by both the number of MTMR antennas and that of the subcarriers.

This paper is organised as follows. Section II describes the system investigated, followed by the correct detection and false alarm probability analysis of both differentially coherent and non-coherent code acquisition schemes in the context of uncorrelated Rayleigh channels in Section III. The MAT expressions of code acquisition designed for MTMR 
antenna assisted MC-DS-CDMA systems are introduced in Section IV. In Section V, our numerical MAT results are discussed, while our conclusions are detailed in Section VI.

\section{System description}

The transmitted signal of the base station having $P$ transmit antennas and $U$ subcarriers can be expressed as

$$
S_{\text {tot }}(t)=\sum_{m=1}^{P} \sum_{u=1}^{U}\left[\sqrt{\frac{E_{\mathrm{c}}}{P T_{\mathrm{c}}}} b(t) c(t) w_{m}(t) \cdot \exp \left(2 \pi f_{u} t+\phi_{u}\right)\right]
$$

where $m=1, \ldots, P$ denotes the number of transmit antennas, $u=1, \ldots, U$ indicates the number of subcarriers, $b(t)$ represents the pilot data sequence assuming a value of binary ' 1 ' [14], $c(t)$ is a common PN sequence having a cell-specific code-phase offset, $w_{m}(t)$ identifies the specific Walsh code assigned to the $p$ th transmit antenna, $E_{\mathrm{c}}$ represents the pilot signal energy per PN code chip, $T_{\mathrm{c}}$ denotes the chip duration, $f_{u}$ denotes the $u$ th subcarrier frequency and $\phi_{u}$ is the $u$ th subcarrier phase of the modulator. In the MC-DS-CDMA system considered, the input bit sequence is serial-to-parallel (SP) converted and each of the parallel sequences is then transmitted on a separate subcarrier. Furthermore, $T_{\mathrm{b}}$ indicates the bit duration of the data sequence before SP conversion, while $T_{\mathrm{s}}$ represents the symbol duration after SP conversion. Consequently, we have $T_{\mathrm{s}}=U \cdot T_{\mathrm{b}}$. The total allocated power is equally shared by the $P$ transmit antennas and $S F=T_{\mathrm{s}} / T_{\mathrm{c}}$ denotes the spreading factor of the subcarrier signals in the MC-DS-CDMA system, while the spreading factor of a corresponding identical-bandwidth SC-DS-CDMA system is $S F_{1}=T_{\mathrm{b}} / T_{\mathrm{c} 1}$, where $T_{\mathrm{c} 1}$ represents the chip duration of the corresponding SC-DS-CDMA signal. For simplicity, in our forthcoming discourse, we assume that there is no overlap between the main spectral lobes of two adjacent subcarriers in the MC-DS-CDMA system considered here [8]. Furthermore, we postulate that each subcarrier signal occupies an identical bandwidth and the total bandwidth is equally divided among the $U$ number of subcarriers. Hence, the relationships of $T_{\mathrm{c}}=U \cdot T_{\mathrm{c} 1}$ and $S F_{1}=S F$ hold, since we have $T_{\mathrm{s}}=U \cdot T_{\mathrm{b}}$. Based upon the above assumption, both the MC- and the corresponding SC-DS-CDMA systems maintain an indentical bandwidth of $2 / T_{\mathrm{c} 1}$, as proposed in [8], which allows their direct comparison in our forthcoming analysis.

The received signal of the MIMO-assisted MC-DSCDMA downlink may be written as

$$
\begin{aligned}
r_{t o t}(t) & =\sum_{m=1}^{P} \sum_{n=1}^{R} \sum_{u=1}^{U} \\
\times & {\left[\begin{array}{c}
\alpha_{(m, n, u)} \sqrt{\frac{E_{\mathrm{c}}}{P T_{\mathrm{c}}}} b\left(t+d T_{c}\right) c\left(t+d T_{\mathrm{c}}\right) w_{m}\left(t+d T_{\mathrm{c}}\right) \\
\cdot \exp \left(2 \pi f_{u} t+\phi_{(m, n, u)}\right)+I_{(m, n, u)}(\mathrm{t})
\end{array}\right] }
\end{aligned}
$$

where $n=1, \ldots, R$ denotes the number of receive antennas, $\alpha_{(m, n, u)}$ is the envelope of the $(m, n, u)$ th received signal path obeying the Rayleigh distribution, $d$ indicates the code phase offset with respect to the phase of the local code. Furthermore, $I_{(m, n, u)}(t)$ is the complex-valued additive white Gaussian noise (AWGN) having a double-sided power spectral density of $I_{0}$, which contaminates the $(m, n, u)$ th path. Fig. 1 portrays either the differentially coherent (DC) or the non-coherent (NC) receiver's schematic designed for

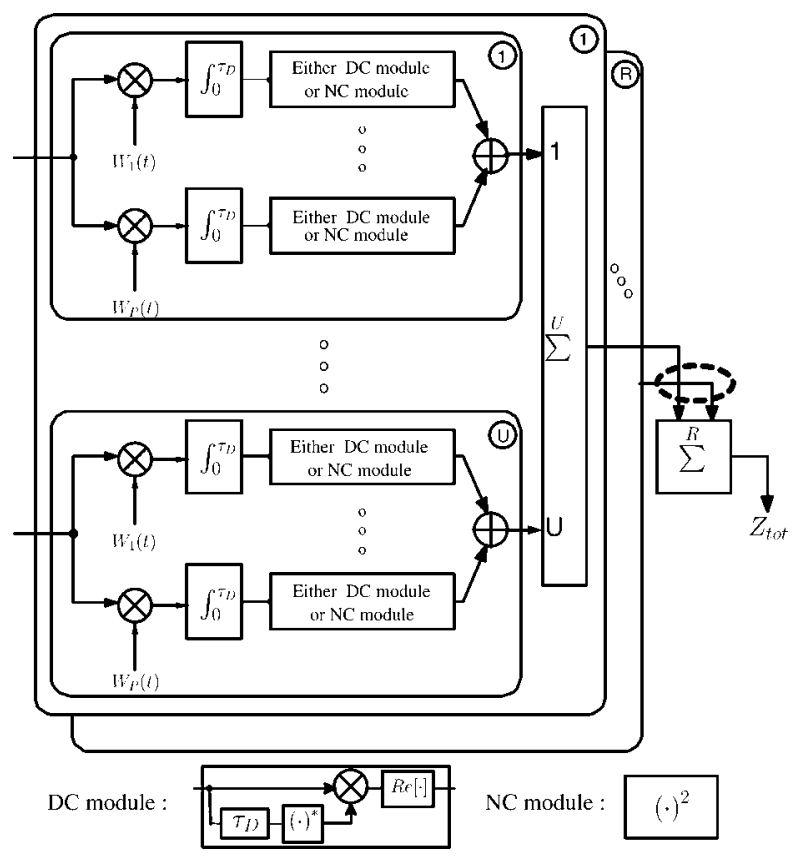

Fig. 1 Receiver structure of either a differentially coherent or a non-coherent code acquisition scheme employing both $R$ receive antennas and U subcarriers

our MC-DS-CDMA code acquisition scheme using MTMR antennas. The receiver generates the decision variable by accumulating $(P R U)$ number of independently faded signals observed over a time interval. In Fig. 1, the numbers 1 to $R$ surrounded by a circle situated in the upper right-hand corner of the largest box represent a specific receiver branch for each receive antenna. Similarly, the numbers 1 to $U$ surrounded by a circle situated in the upper right-hand corner of the smaller inner box represent a decoding module dedicated to each of the subcarriers. In order to simplify the receiver's structure, we omitted the front-end down converter, chip-matched filter, sampler and descrambler of the PN code. Further details on the related schemes may be found in [12]. In the DC scheme of Fig. 1, instead of squaring the summed energy as suggested by the procedures outlined in $[10,14]$, the channel's output samples accumulated over a full spreading code period are multiplied by the conjugate of the $N$-chip-delayed samples $[11,12]$, where $N$ indicates the number of chips accumulated over the integral dwell time $\tau_{D}$.

When analysing the MAT performance of the DDSS technique [15], the DC scheme of Fig. 1 is used in the verification mode. The employment of DC detection becomes feasible, since the code acquisition was successfully accomplished. This is because the DC scheme of Fig. 1 is capable of enhancing the performance of a DDSS system by increasing the reliability of a decision variable in the verification mode, as suggested in [13]. In contrast, the NC structure of Fig. 1 is employed in the search mode preceding the verification mode, that is before the desired user's code was acquired. For the sake of comparison, the NC counterpart of a DC DDSS system obeys the NC structure of Fig. 1 in both of its search and verification mode. For the sake of highlighting the overall structure of our DDSS code acqusition systems, Fig. 2 portrays the flow chart of the system, where $\theta_{1}$ and $\theta_{2}$ represent the threshold of the search and verification mode, respectively. Furthermore, $Z_{1}$ and $Z_{2}$ denote the decision variables of the search and verification mode, respectively. Further details on the related DDSS system are found in [13]. 


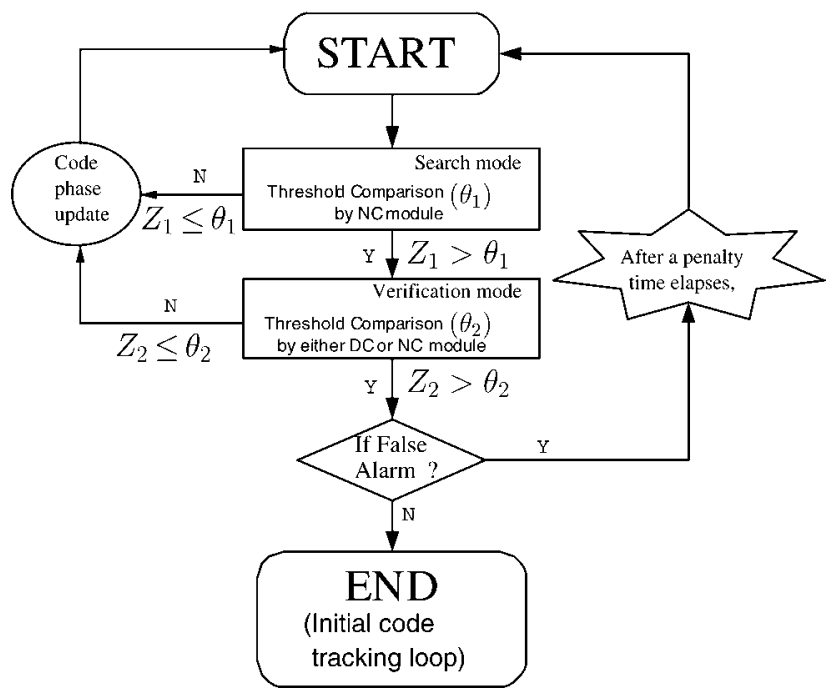

Fig. 2 Flow chart of our DDSS code acquisition scheme

\section{Theoretical analysis of both differentially coherent and non-coherent code acquisition}

\subsection{Correct detection and false alarm probability of the differentially coherent code acquisition scheme}

Again, the decision variable is generated by accumulating $(P R U)$ number of independently faded received signals observed over a time interval in order to improve the correct detection probability, when the mobile channel imposes both fading and poor SINR conditions, where $P$, $R$ and $U$ are the number of transmit and receive antennas as well as subcarriers, respectively. Here we omitted formulating the final decision variable, which may be readily derived using the procedures proposed in [12] in the context of the receiver structure of Fig. 1. The final DC decision variable may be expressed as [12]

$$
\begin{aligned}
Z_{k}^{d c} & =\sum_{m=1}^{P} \sum_{n=1}^{R} \sum_{u=1}^{U} \\
& \times\left[\left(\sqrt{\frac{4 E_{\mathrm{c}}}{N I_{0} P}} \cdot S_{k(m, n, u)}+W_{1, k(m, n, u)}\right)^{2}+W_{3, k(m, n, u)}^{2}\right] \\
& -\sum_{m=1}^{P} \sum_{n=1}^{R} \sum_{u=1}^{U}\left[W_{2, k(m, n, u)}^{2}+W_{4, k(m, n, u)}^{2}\right]
\end{aligned}
$$

where $k$ indicates the $k$ th chip's sampling instant, $S_{k(m, n, u)}$ is assumed to be deterministic [12] and the definition of $W_{1, k(m, n, u)}, W_{2, k(m, n, u)}, W_{3, k(m, n, u)}$ and $W_{4, k(m, n, u)}$ is the same as in [12]. These are mutually independent Gaussian random variables having zero means and unit variances [12]. Let us now introduce a shorthand for the first and second terms of (3) as follows

$$
\begin{aligned}
& X_{k}=\sum_{m=1}^{P} \sum_{n=1}^{R} \sum_{u=1}^{U} \\
& \times\left[\left(\sqrt{\frac{4 E_{c}}{N I_{0} P}} \cdot S_{k(m, n, u)}+W_{1, k(m, n, u)}\right)^{2}+W_{3, k(m, n, u)}^{2}\right]
\end{aligned}
$$

and

$$
Y_{k}=\sum_{m=1}^{P} \sum_{n=1}^{R} \sum_{u=1}^{U}\left[W_{2, k(m, n, u)}^{2}+W_{4, k(m, n, u)}^{2}\right]
$$

Then the final decision variable of (3) is expressed as $\quad Z_{k}^{d c}=X_{k}-Y_{k}=\sum_{m=1}^{P} \sum_{n=1}^{R} \sum_{u=1}^{U} X_{k(m, n, u)}-\sum_{m=1}^{P}$ $\sum_{n=1}^{R} \sum_{u=1}^{U} Y_{k(m, n, u)}$, where $X_{k}$ is non-centrally chi-square distributed with $(2 P \cdot R \cdot U)$ degrees of freedom and its noncentrality parameter $\lambda_{x}$ is either $4 N / P\left(E_{\mathrm{c}} / I_{0}\right)^{\prime}$ for the hypothesis of the desired signal being present $(x=1)$ or $4 / N P\left(E_{\mathrm{c}} / I_{0}\right)^{\prime}$ for it being absent $(x=0)$ [12], whereas $Y_{k}$ follows a central chi-square PDF with $(2 P R U)$ degrees of freedom. In the spirit of [14], the definition of $\left(E_{\mathrm{c}} / I_{0}\right)^{\prime}$ encapsulates the effects of both timing errors and frequency mismatches. Accordingly, the PDF of $X_{k}$ and $Y_{k}$ can be expressed as [16]

$$
\begin{aligned}
f_{X_{k}}\left(z \mid H_{x}\right)= & \frac{1}{2}\left(\frac{z}{\lambda_{x}}\right)^{(P R U-1) / 2} \\
& \cdot \exp \left[-\frac{\left(z+\lambda_{x}\right)}{2}\right] \cdot \mathcal{I}_{(P R U-1)}\left(\sqrt{z \cdot \lambda_{x}}\right)
\end{aligned}
$$

and

$$
f_{Y_{k}}\left(z \mid H_{x}\right)=\frac{1}{2^{P R U} \cdot \Gamma(P R U)} \cdot z^{(P R U-1)} \cdot \exp \left[-\frac{z}{2}\right]
$$

respectively, where we have $z \geq 0, x=0$ or $1, \Gamma(\cdot)$ denotes the Gamma function and $\mathcal{I}_{(P R U-1)}(\cdot)$ is the $(P R U-1)$ st-order modified Bessel function.

Our objective is now that of deriving the PDF of the desired user's signal conditioned on the presence of the desired signal in $f_{X_{k}}\left(z \mid H_{x}\right)$ formulated for transmission over an uncorrelated Rayleigh channel. In this scenario, $E_{\mathrm{c}}$ is multiplied by the square of the Rayleigh-distributed fading amplitude, $\beta$, which exhibits a chi-square distribution having two degrees of freedom and it is hence expressed as $p(\beta)=e^{-\beta / \sigma^{2}} / \sigma^{2}$, where $\sigma^{2}$ is the variance of the constituent Gaussian distribution. Then the average pilot signal energy $\bar{E}_{\mathrm{c}}$ per PN code chip can be expressed as $\bar{E}_{\mathrm{c}}=\bar{\beta} E_{\mathrm{c}}=\sigma^{2} E_{\mathrm{c}}$. Hence first the PDF $f_{Z_{\left.Z_{k(m, n, u}\right)}}\left(z \mid H_{x}, \beta\right)$ corresponding to $\beta$ conditioned on the hypothesis of the desired signal being transmitted over an AWGN channel having this specific SINR is weighted by the probability of occurrence $f(\beta)$ of encountering $\beta$, as quantified by the PDF. The resultant product is then averaged over its legitimate range of $-\infty \sim \infty$, yielding

$$
\begin{aligned}
f_{X_{k(m, n, u)}}\left(z \mid H_{x}\right)= & \int_{-\infty}^{\infty} f(\beta) \cdot f_{X_{k(m, n, u)}}\left(z \mid H_{x}, \beta\right) \mathrm{d} \beta \\
= & \int_{0}^{\infty}\left(\frac{\mathrm{e}^{-\beta / \sigma^{2}}}{\sigma^{2}}\right) \cdot \frac{\exp \left[-\left(z+\beta \lambda_{x}\right) / 2\right]}{2} \\
& \cdot \mathcal{I}_{0}\left(\sqrt{\beta \lambda_{x} z}\right) \mathrm{d} \beta \\
= & \frac{\exp \left[-z /\left(2+\lambda_{x} \sigma^{2}\right)\right]}{\left(2+\lambda_{x} \sigma^{2}\right)} \\
\equiv & \frac{\exp \left[-z /\left(2+\bar{\lambda}_{x}\right)\right]}{\left(2+\bar{\lambda}_{x}\right)}
\end{aligned}
$$

where the corresponding non-centrality parameter of $\bar{\lambda}_{x} \equiv \lambda_{x} \sigma^{2}$ is either $4 N / P\left(\bar{E}_{\mathrm{c}} / I_{0}\right)^{\prime}$ when the desired signal is deemed to be present $(x=1)$ or $4 / N P\left(\bar{E}_{\mathrm{c}} / I_{0}\right)^{\prime}$ when it is deemed to be absent $(x=0)$. For notational convenience, we also define a new biased non-centrality parameter 
$\mu_{x}=\left(2+\bar{\lambda}_{x}\right)$. Further details on the related derivations are provided in $[12,14]$. Finally, we arrive at the PDF of $X_{k(m, n, u)}$ conditioned on the presence of the desired signal in the form of

$$
f_{X_{k(m, n, u)}}\left(z \mid H_{x}\right)=\frac{1}{\mu_{x}} \mathrm{e}^{-z / \mu_{x}}
$$

In contrast, the PDF of $Y_{k(m, n, u)}$ may be readily derived from (7), leading to

$$
f_{Y_{k(m, n, u)}}\left(z \mid H_{x}\right)=\frac{1}{2} \mathrm{e}^{-z / 2}
$$

Since the decision variables, $X_{k}$ and $Y_{k}$ are expressed as the sum of (PRU) number of independent variables $\left(X_{k}=\sum_{m=1}^{P} \sum_{n=1}^{R} \sum_{u=1}^{U} X_{k(m, n, u)} \quad\right.$ and $Y_{k}=$ $\left.\sum_{m=1}^{P} \sum_{n=1}^{R} \sum_{u=1}^{U} Y_{k(m, n, u)}\right)$, each of which obeys a PDF given by either (12) or (13), we can determine the Laplace transform of each by raising them to the $(P R U)$ th power and then performing the inverse transform in order to generate the desired PDF [14], yielding

$$
\begin{aligned}
& f_{X_{k}}\left(z \mid H_{x}\right)=\frac{z^{(P R U-1)} \mathrm{e}^{-z / \mu_{x}}}{\Gamma(P R U) \cdot \mu_{x}^{P R U}} \\
& f_{Y_{k}}\left(z \mid H_{x}\right)=\frac{z^{(P R U-1)} \mathrm{e}^{-z / 2}}{\Gamma(P R U) \cdot 2^{P R U}}
\end{aligned}
$$

Then, the PDF of $Z_{k}^{d c}=X_{k}-Y_{k}$ can be calculated by straightforward convolution of the PDFs of both $X_{k}$ and $Y_{k}$. This leads to the PDF of the difference between two independent Gamma variables. To elaborate a little further, we have $X_{\text {tot }} \sim g\left(P R U, \mu_{x}\right)$ and $Y_{\text {tot }} \sim g(P R U$, $2)$, where this shorthand represents that both $X_{\text {tot }}$ and $Y_{\text {tot }}$ obey a Gamma distribution having the shape parameter of $(P R U)$ and the scale parameter of either $\mu_{x}$ or 2 , respectively, as outlined in [17]. The convolution of the PDFs $f_{X_{k}}$ and $f_{Y_{k}}$ formulated in an effort to compute the PDF of $Z_{k}^{d c}$ conditioned on the desired signal being present or absent is derived as [17]

$$
\begin{aligned}
f_{Z_{k}^{d c}}\left(z \mid H_{x}\right)= & \int_{-\infty}^{\infty} f_{X_{k}}(\xi) \cdot f_{Y_{k}}(\xi-z) \mathrm{d} \xi \\
= & \left(\frac{\left(1-c^{2}\right)^{a+(1 / 2)} \cdot|z|^{a}}{\sqrt{\pi} \cdot 2^{a} \cdot b^{a+1} \cdot \Gamma(a+(1 / 2))}\right) \\
& \cdot \exp \left(-\frac{c}{b} z\right) \cdot K_{a}\left(\frac{|z|}{b}\right), \quad z \neq 0
\end{aligned}
$$

where $K_{a}(\cdot)$ indicates the modified Bessel function of the second kind and of order $a$. We note furthermore that $K_{a}(\cdot)$ is undefined, when the argument is equal to zero. However, this fact has a negligible impact on calculating the probability of correct detection and false alarm. Further details on the associated statements are found in [17]. Let us now define the three parameters, namely $a=P R U-0.5, \quad b=\left(4 \mu_{x}\right) /\left(\mu_{x}+2\right) \quad$ and $c=-\left(\mu_{x}-2\right) /\left(\mu_{x}+2\right)$, which allow us to express the probability of correct detection or false alarm according to $x=1$ or 0 , respectively, as follows [17]

$$
\begin{aligned}
\left.P^{d c}\right|_{x=1 \text { or } 0}= & \int_{\theta}^{\infty}\left(\frac{\left(1-c^{2}\right)^{a+(1 / 2)} \cdot|z|^{a}}{\sqrt{\pi} \cdot 2^{a} \cdot b^{a+1} \cdot \Gamma(a+(1 / 2))}\right) \\
& \left.\cdot \exp \left(-\frac{c}{b} z\right) \cdot K_{a}\left(\frac{|z|}{b}\right) \mathrm{d} z\right|_{x=1 \text { or } 0}, \theta \neq 0
\end{aligned}
$$

where $\theta$ is a threshold value.

\subsection{Correct detection and false alarm probability of the non-coherent code acquisition scheme}

For comparison, the NC counterpart of the previously described DC scheme is analysed here, where the final decision variable is given by [12].

$$
Z_{k}^{n c}=\sum_{m=1}^{P} \sum_{n=1}^{R} \sum_{u=1}^{U}\left\|\frac{1}{\sqrt{2}} \cdot\left(\sqrt{\frac{4 E_{\mathrm{c}}}{N I_{0} P}} \cdot S_{k(m, n, u)}+I_{(m, n, u)}\right)\right\|^{2}
$$

where $\|\cdot\|^{2}$ denotes the squared envelope of the complexvalued argument and $1 / \sqrt{2}$ is a normalisation factor for the noise variance. Accordingly, the NC decision variable $Z_{k}^{n c}$ follows a non-central chi-square PDF with (2PRU) degrees of freedom and its non-centrality parameter $\lambda_{x}$ is either $2 N / P\left(E_{\mathrm{c}} / I_{0}\right)^{\prime}$ for the hypothesis of the desired signal being present $(x=1)$ or $2 / N P\left(E_{\mathrm{c}} / I_{0}\right)^{\prime}$ for it being absent $(x=0)$ [12], where the PDF is given by [16].

$$
\begin{aligned}
f_{Z_{k}^{n c}}\left(z \mid H_{x}\right)= & \frac{1}{2}\left(\frac{z}{\lambda_{x}}\right)^{(P R U-1) / 2} \\
& \cdot \exp \left[-\frac{\left(z+\lambda_{x}\right)}{2}\right] \cdot \mathcal{I}_{(P R U-1)}\left(\sqrt{z \cdot \lambda_{x}}\right)
\end{aligned}
$$

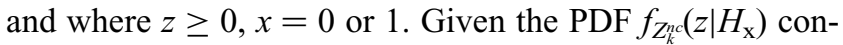
ditioned on the presence of the desired signal, which was formulated for transmission over an uncorrelated Rayleigh channel, and following a similar procedure to that outlined in Section 3.1, finally, the probability of correct detection or false alarm corresponding to $x=1$ or 0 , respectively is expressed as

$$
\left.P^{n c}\right|_{x=1 \text { or } 0}=\left.\exp \left(-\frac{\theta}{\mu_{x}}\right) \cdot \sum_{k=0}^{P R U-1} \frac{\left(\theta / \mu_{x}\right)^{k}}{k !}\right|_{x=1 \text { or } 0}
$$

where $\mu_{\mathrm{x}}$ is again set to be $\left(2+\bar{\lambda}_{x}\right)$ and $\bar{\lambda}_{x}$ is either $2 / N P\left(\bar{E}_{\mathrm{c}} / I_{0}\right)^{\prime}$, when the desired signal is deemed to be present $(x=1)$ or $2 N / P\left(\bar{E}_{\mathrm{c}} / I_{0}\right)^{\prime}$ when it is deemed to be absent $(x=0)$. Given (18) and (21), the transfer functions required for characterising the multiple antenna assisted SC- and MC-DS-CDMA schemes can be readily derived $[14,15]$.

Before concluding this section, it is worth noting that the PN sequence' chip duration $T_{\mathrm{c} 1}$ of the SC-DS-CDMA system is $U$ times lower than that of the MC-DS-CDMA arrangement, namely, we have $T_{\mathrm{c} 1}=T_{c} / U$. This is because given the same allocated bandwidth and the same total transmitted energy per chip, the bandwidth of the SC-DS-CDMA signal is $U$ times higher than that of the subcarrier signals in the MC-DS-CDMA system using $U$ subcarriers. Moreover, in an effort to maintain a constant integral dwell time of $\tau_{\mathrm{D}}$, the chip energy summed by the SC-DS-CDMA receiver during the period of $\tau_{\mathrm{D}}$ is $U$ times higher than that collected by the MC-DS-CDMA correlator of each subcarrier, since the number of chips within the period of $\tau_{\mathrm{D}}$ is $U$ times higher for the SC-DS-CDMA system than that of the MC-DS-CDMA system [8].

\section{MAT analysis of code acquisition}

Classically, serial search techniques $[6,14,15]$ have been adopted in detection scenarios, when the uncertainty region is quite wide, for example in the order of $\left(2^{15}-1\right)$ chips, as in the downlink of the inter-cell synchronous CDMA-2000 system [14, 15]. The main design objective of the family of serial search techniques is to achieve as 
low an MAT as possible. Explicit MAT formulae derived for a single-antenna assisted serial search-based code acquisition arrangement were provided in [14] for single dwell serial search and [15] for DDSS, respectively. Code acquisition schemes designed for both SC-DS-CDMA and MC-DS-CDMA play a pivotal role in synchronising with the direct line-of-sight received signal path, corresponding to the reference finger of the Rake receiver. There is no difference between a single-antenna and a multiple-antenna assisted MC-DS-CDMA system in terms of characterising their MAT performance, except for the derivation of the correct detection and the false alarm probability as a function of both the number of MTMR antennas and that of the subcarriers, both of which have a dominant impact on the attainable MAT. We will continue our discourse here by comparing the MAT performance of a DC code acquisition scheme and its NC counterpart employing DDSS $[11,13,15]$.

We postulate that in each chip duration $T_{\mathrm{c}}, l$ number of correct timing hypotheses are examined, which are spaced by $T_{\mathrm{c}} / l$. Hence the entire uncertainty region is increased $l$-fold. All the resultant $(v-2 l)$ number of states that may lead to a false alarm are expected to increase the MAT proportionately to the related penalty time. The penalty time is defined as the time required for the system to return to its search mode after mistakenly deciding that correct phase-acquisition was achieved, although it was not. The $2 l$ legitimate locking states within a single chip duration of the correct timing instant are taken into account in the MAT analysis. Combining all these $2 l$ legitimate locking states according to either (18) or (21) into the correct detection transfer function defined in [15] leads to the correct detection transfer function expressed as

$$
\begin{aligned}
H_{\mathrm{D}}(z)= & \sum_{j=1}^{2 l} P_{1 j}^{(1)} P_{1 j}^{(2)} z^{m+1} \\
& \times \prod_{i=1}^{j-1}\left[\left(1-P_{1 i}^{(1)}\right) z+P_{1 i}^{(1)}\left(1-P_{1 i}^{(2)}\right) z^{m+1}\right]
\end{aligned}
$$

where $P_{1 j}^{(1)}$ and $P_{1 j}^{(2)}$ represent the correct detection probability associated with the $j$ th correct detection of the first and second dwell intervals, respectively, following $(j-1)$ previous trials each resulting in a miss and where each probability of a miss in the corresponding total miss transfer function is given by $\left(1-P_{1 i}^{(1)}\right)$ or $P_{1 i}^{(1)}\left(1-P_{1 i}^{(2)}\right)$. Furthermore, $z$ indicates the unit-delay operator and $m$ represents the exponent of $z$ in the verification mode and $H_{0}(z)$ denotes the absence of the desired user's signal at the output of the acquisition scheme, which is expressed as

$$
\begin{aligned}
\tilde{H}_{0}(z)= & \left(1-P_{0}^{(1)}\right) z+P_{0}^{(1)}\left(1-P_{0}^{(2)}\right) z^{m+1} \\
& +P_{0}^{(1)} P_{0}^{(2)} z^{K+m+1}
\end{aligned}
$$

where $P_{0}^{(1)}$ and $P_{0}^{(2)}$ represent the false alarm probability of the first and second dwell intervals, respectively, and $K$ is the false locking penalty factor defined in [14]. Finally, $H_{\mathrm{M}}(z)$ represents the overall miss probability of a search run carried out across the entire uncertainty region, which may be formulated as the product of the summation of the two individual miss probabilities, since these may be considered independent events, yielding

$$
H_{\mathrm{M}}(z)=\prod_{j=1}^{2 l}\left[\left(1-P_{1 j}^{(1)}\right) z+P_{1 j}^{(1)}\left(1-P_{1 j}^{(2)}\right) z^{m+1}\right]
$$

Then, it may be shown that the generalised expression formulated for calculating the MAT of the serial search-based code acquisition scheme is given by $[14,15]$.

$$
\begin{aligned}
& E\left[T_{\mathrm{ACQ}}\right]=\frac{1}{H_{\mathrm{D}} 1}\left[H_{\mathrm{D}}^{\prime}(1)+H_{\mathrm{M}}^{\prime}(1)\right. \\
& \left.+\left\{(v-2 l)\left[1-\frac{H_{\mathrm{D}}(1)}{2}\right]+\frac{1}{2} H_{\mathrm{D}}(1)\right\} H_{0}^{\prime}(1)\right] \cdot \tau_{D 1}
\end{aligned}
$$

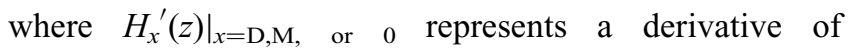
$\left.H_{x}(z)\right|_{x=D, M, \text { or } 0}$ and $\tau_{D I}$ indicates the first dwell time.

Upon substituting (18) and (21) into the appropriate transfer functions given in (22)-(24) and then the resultant formulae into (25) for the sake of characterising the MIMO-aided SC- and MC-DS-CDMA schemes leads to the corresponding MAT results.

\section{Numerical system performance results}

In this section, the numerical MAT performance of the MTMR antenna assisted MC-DS-CDMA code acquisition scheme of Fig. 1 will be characterised. Our performance comparison between the SC-DS-CDMA system $(U=1)$ and the MC-DS-CDMA system using $U=4$ subcarriers is based upon the assumptions that these systems have the same total transmitted energy per chip. Furthermore, it is assumed that the integral dwell time, $\tau_{\mathrm{D}}$, is the same for all the scenarios considered here. The associated system parameters are summarised in Table 1. In Table 2, we summarised the maximum SINR degradation inflicted by both the Doppler shift and the frequency mismatch between the transmitter and receiver in conjunction with the coherent

\section{Table 1: System parameters}

\begin{tabular}{ll}
\hline Bandwidth, $\mathrm{MHz}$ & \\
$\quad \begin{array}{l}U=1 \\
U=4\end{array}$ & 2.4576 \\
Carrier frequency, $\mathrm{GHZ}$ & 1.9 \\
Spreading factory & 128 \\
Diversity & \\
$\quad$ Transmit & $1,2,4$ \\
Receive & $1,2,4$ \\
Subcarrier & 1,4 \\
Frequency mismatch, $\mathrm{Hz}$ & 1000 \\
Mobile speed, km/h & 160 \\
Number. of chip & \\
$\quad U=1$ & 128 and 512 chips \\
$\quad U=4$ & 32 and 128 chips \\
Total uncertainty region & 512 \\
False locking penalty factor & 1000 \\
\hline
\end{tabular}

Table 2: Maximum SINR degradation inflicted by both the Doppler shift and a $1000 \mathrm{~Hz}$ frequency mismatch in comparison to a stationary receiver having no frequency drift for the coherent integration interval of $\boldsymbol{N}$ chip durations at a carrier frequency of $1.9 \mathrm{GHz}$ as a function of the number of subcarriers $(U=1$ and 4$)$

\begin{tabular}{llllll}
\hline$N($ Chips $): U=1$ & 128 & 256 & 512 & 768 & 1024 \\
$N($ Chips): $U=4$ & 32 & 64 & 128 & 192 & 256 \\
Degradation, dB & 0.061 & 0.2449 & 0.9969 & 2.3144 & 4.3213 \\
\hline
\end{tabular}


integration interval of $\tau_{\mathrm{D}}$ duration, as seen in Fig. 1. It was assumed that the length of the PN sequence in our system was $512 T_{\mathrm{cl} 1}$ (or $128 T_{\mathrm{c}}$ ), where the chip-durations chosen for $U=1$ and 4 are $T_{\mathrm{c} 1}=1 / 2.4576 \mu \mathrm{s}$ and $T_{\mathrm{c}}=1 / 0.6144 \mu s$, respectively. The coherent accumulator of the DC scheme seen in Fig. 1 adopted the FPC scheme of [12] in the verification mode of the DDSS scheme in order to improve the attainable MAT performance, where the number of chips over which the accumulator $\sum$ sums the $(\cdot)^{2}$ envelope detector's output in both the search and the verification modes of DDSS are assumed to be 128 and 512 chips in the SC scheme of $U=1,32$ and 128 chips in the $U=4$ MC-DS-CDMA arrangement, respectively. These optimised parameter values were computed by using (18), (21) and (25) as well as (3.7) of [14], which were provided for quantifying the performance degradation imposed by both the Doppler shift and the frequency mismatch encountered. The spreading factor of the Walsh code to be acquired was chosen to be 128 . The carrier frequency was $1.9 \mathrm{GHz}$ and the frequency mismatch was postulated to be $1000 \mathrm{~Hz}$ [14]. As a worst-case mobile speed, it is reasonable to assume $160 \mathrm{~km} / \mathrm{h}$. We also assumed that the sampling inaccuracy caused by having a finite, rather than infinitesimally low search step size of $\Delta=1 / 2 T_{\mathrm{c}}$ was $-0.91 \mathrm{~dB}$, which is a practically acceptable value for the specific search step size considered [14]. The entire uncertainty region of code acquisition was assumed to entail 512 hypotheses. This value is the uncertainty region of the $U=2$ scenario, because the same uncertainty region is required for the sake of a fair comparison. Finally, in the spirit of [18], the false locking penalty factor was considered to be 1000 . For simplicity, it was assumed that only a single received signal path is encountered in a given search window. All the performance curves have been generated at the threshold value of $E_{\mathrm{c}} / I_{\mathrm{o}}=-19 \mathrm{~d} B$. This was experimentally found for the specific code acquisition scheme considered.

Fig. 3 illustrates the achievable MAT against SINR per chip performance of the DDSS-aided SC-DS-CDMA code acquisition arrangement. More specifically, both the NC code acquisition assisted scheme used in the search mode and the DC code acquisition assisted arrangement employed

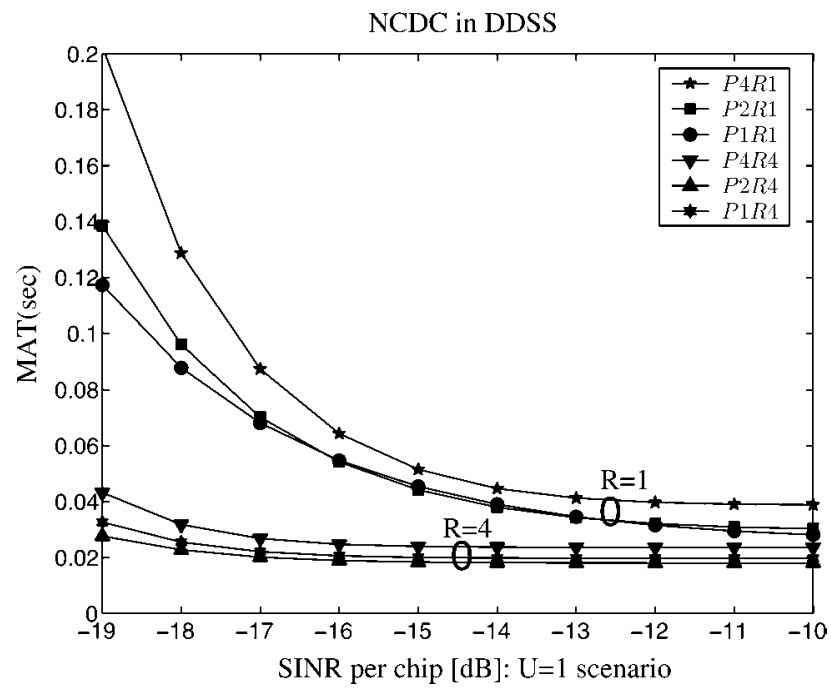

Fig. 3 MAT against SINR per chip performance for a DDSS scheme constituted by both the non-coherent code acquisition assisted scheme in the search mode and the differentially coherent code acquisition scheme in the verification mode parameterised with both the number of transmit and receive antennas for ' $U=1$ ' subcarrier

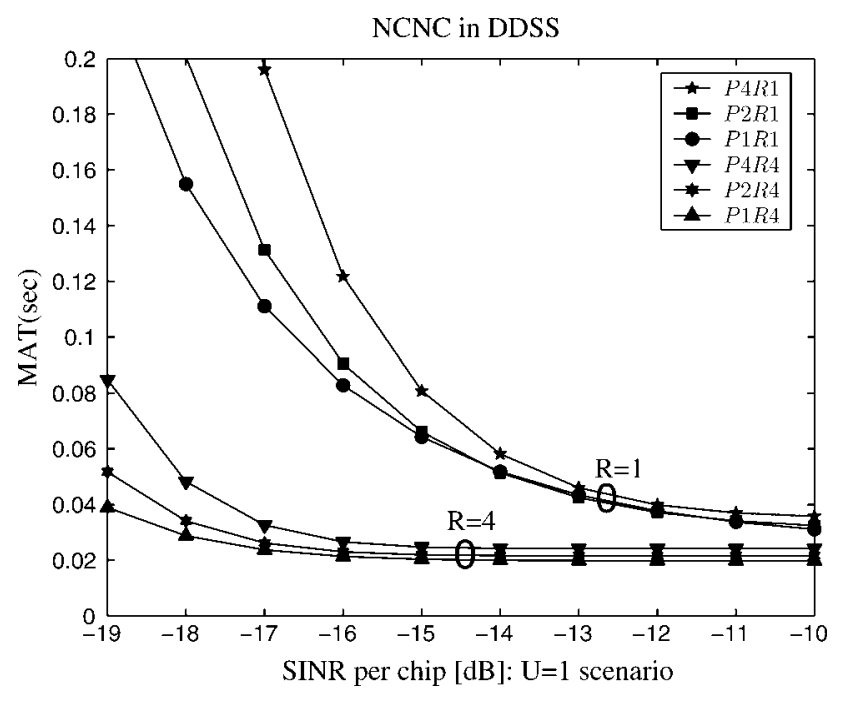

Fig. 4 MAT against SINR per chip performance of the DDSS-assisted non-coherent code acquisition scheme parameterised with both the number of transmit and receive antennas for ' $U=1$ ' subcarrier

in the verification mode are characterised as a function of both the number of transmit antennas for $P=1,2$ as well as 4 and that of the number of receive antennas for $R=1$ and 4 . In contrast, Fig. 4 characterises the MAT against SINR per chip performance of the DDSS-aided NC code acquisition scheme, both in its search mode and verification mode. Observe in Fig. 3 that unexpectedly, as the number of transmit antennas is decreased, all the curves explicitly indicate an improved MAT performance, except for the ' $P 2 R 1$ ' scenario, as recorded for the specific SINR range between -13 and $-16 \mathrm{~dB}$. To elaborate on the above observation a little further, in the scenario of ' $P 2 R 1$ ' the DDSS scheme exhibits a slightly better MAT peformance in comparison to the ' $P 1 R 1$ ' scenario right across the specific SINR range considered. Similarly, as the number of transmit antennas is decreased in Fig. 4, all the MAT curves illustrate an improved peformance, except for the ' $P 2 R 1$ ' scenario, which constituted an exception also in Fig. 3. The results seen in Fig. 3 suggest that the overall performance improvement of the DDSS-aided DC scheme of Fig. 1 recorded in its verification mode is significantly higher than that of the DDSS-assisted NC scheme of Fig. 1, as seen in Fig. 4. The DC scheme has a performance gain of just under $3 \mathrm{~dB}$ over the $\mathrm{NC}$ arrangement, when considering their correct detection probability and false alarm probability. However, due to using an NC scheme in the search mode, the MAT performance gain of the NCDC scheme of Fig. 3 over that of the NCNC scheme seen in Fig. 4 is around $2 \mathrm{~dB}$. Hence we conclude that the MAT performance curves confirm the expected trends. Moreover, the DC scheme has an advantage over the NC one in the low SINR range [11] in terms of reducing the effects of both the AWGN and interference. In the low-SINR region, the false alarm probability difference between the DC and NC schemes corresponds to a factor of two, indicating the superiority of the DC scheme over the NC one. Hence, this indicates that the MIMO-aided DC scheme experiences a lower MAT peformance degradation owing to the reduced transmit power of the individual transmit antennas than its $\mathrm{NC}$ counterpart. It is worth mentioning that although not shown in Figs. 3 and 4 for reasons of avoiding obfuscating details, the reliable operating range of $R=2$ receive antennas is situated in between that corresponding to the $R=1$ and $R=4$ receive antenna scenario. 


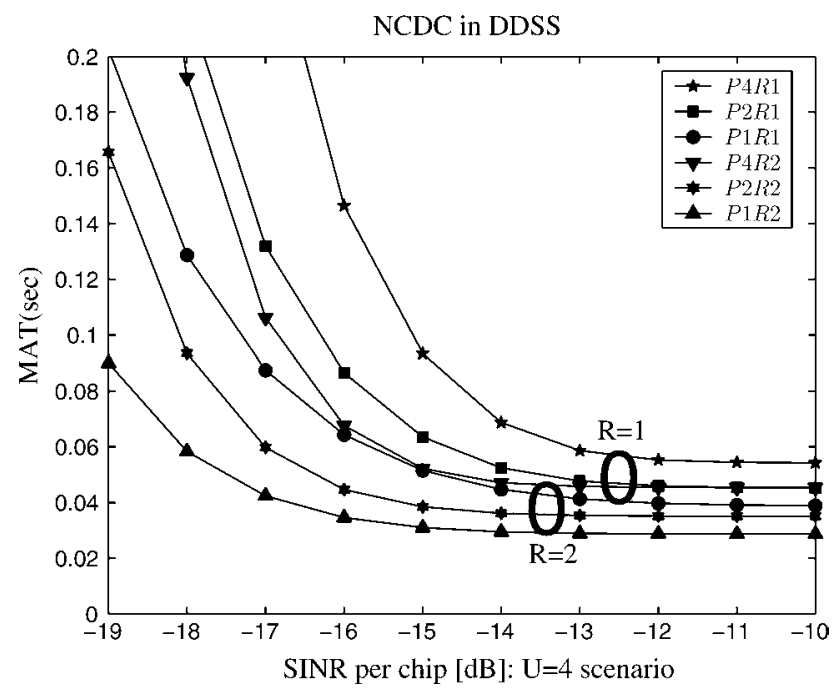

Fig. 5 MAT against SINR per chip performance for a DDSS scheme constituted by both the non-coherent code acquisition assisted scheme in the search mode and the differentially coherent code acquisition scheme in the verification mode parameterised with both the number of transmit and receive antennas for ' $U=4$ ' subcarriers

Fig. 5 illustrates the achievable MAT against SINR per chip performance of the DDSS-assisted MC-DS-CDMA code acquisition scheme using $\mathrm{NC}$ code acquisition in its search mode and DC code acquisition in its verification mode. The MAT results are parameterised with both the number of MTMR antennas for ' $U=4$ ' subcarriers. For the sake of comparison, Fig. 6 characterises the MAT against SINR per chip performance of the DDSS-assisted NC code acquisition scheme in its search mode and verification mode parameterised with both the number of MTMR antennas for ' $U=4$ ' subcarriers. In the case of the MC-DS-CDMA system, both schemes have unnecessarily high-diversity order, which is determined by the number of subcarriers used. It is also assumed that the total transmitted energy per chip is the same in all the scenarios considered. Accordingly, the achievable diversity order is determined by the product of the number of subcarriers and that of the number of transmit antennas, as documented in $[4,8-10]$. This phenomenon indicates that the

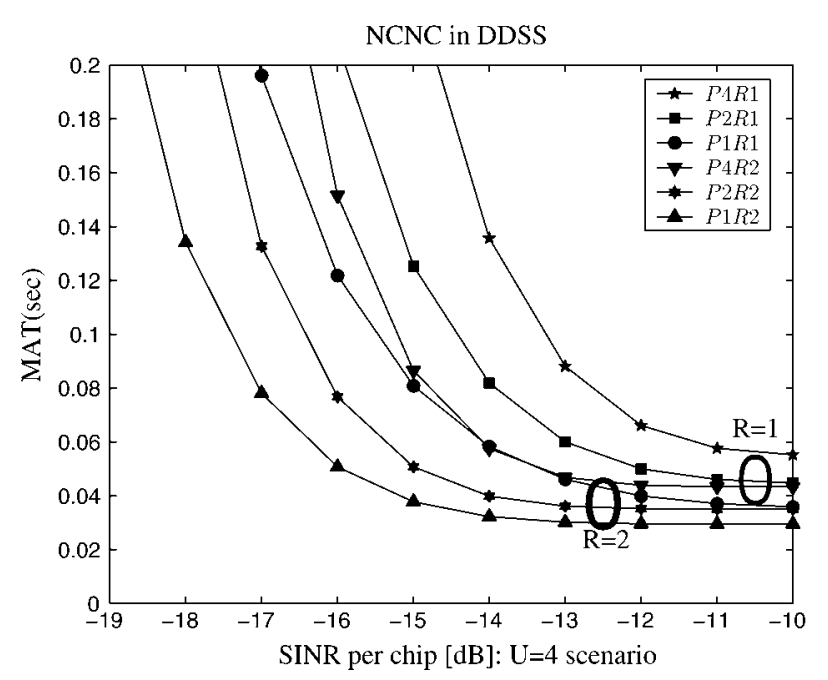

Fig. 6 MAT against SINR per chip performance of the DDSS assisted non-coherent code acquisition scheme parameterised with both the number of transmit and receive antennas for ' $U=4$ ' subcarriers employment of multi-carrier transmissions leads to exactly the same detrimental effect on the achievable MAT performance as that imposed by employing multiple transmit antennas owing to the reduced 'per-diversity-branch' power, as argued in what follows. The results of Figs. 5 and 6 are parameterised by both the number of transmit antennas for $P=1,2$ as well as 4 and by the number of receive antennas for $R=1$ as well as 2. As the number of transmit antennas is decreased, all the curves of Figs. 5 and 6 exhibit an improved MAT peformance. Furthermore, as a benefit of the inherent performance gain of the $\mathrm{DC}$ scheme over the $\mathrm{NC}$ one, the overall MAT peformance results of Fig. 5 are better than those of Fig. 6. Although the results of the DDSS scenario characterised in both Figs. 5 and 6 exhibit a similar trend as in both Figs. 3 and 4, the peformance degradation imposed by employing both multiple antennas and multiple subcarriers becomes more drastic in both Figs. 5 and 6, when the number of transmit antennas is increased. This fact explicitly illustrates that the DDSS-assisted MC-DS-CDMA code acquisition scheme considerably degrades the achievable MAT performance of SC-DS-CDMA. This is the consequence of two phenomena. First, it is imposed by the low per-antenna power imposed by using multiple transmit antennas for the sake of achieving either a transmit diversity gain or a multiplexing gain. The second reason for the reduced MAT performance of MC-DS-CDMA is the low per-subcarrier power imposed by the introduction of multiple subcarriers in order to attain a frequency diversity gain. Nonetheless, we demonstrated that the employment of the DC scheme improves the achievable MAT performance. To elaborate on the above results a little further, a low level of per-branch and/or per-subcarrier received signal strength is expected to result in a low acquisition performance, despite achieving a high transmit-and frequency-diversity gain. In other words, a high transmit-and frequency-diversity order effectively leads to a code acquisition performance loss, as a consequence of the insufficiently high signal strength per transmit antenna and per subcarrier. When employing multiple receive antennas, similar trends are observable, although using multiple receive antennas has the potential of alleviating the acquisition performance degradation imposed by the low per-branch $E_{\mathrm{c}} / I_{\mathrm{o}}$ values due to
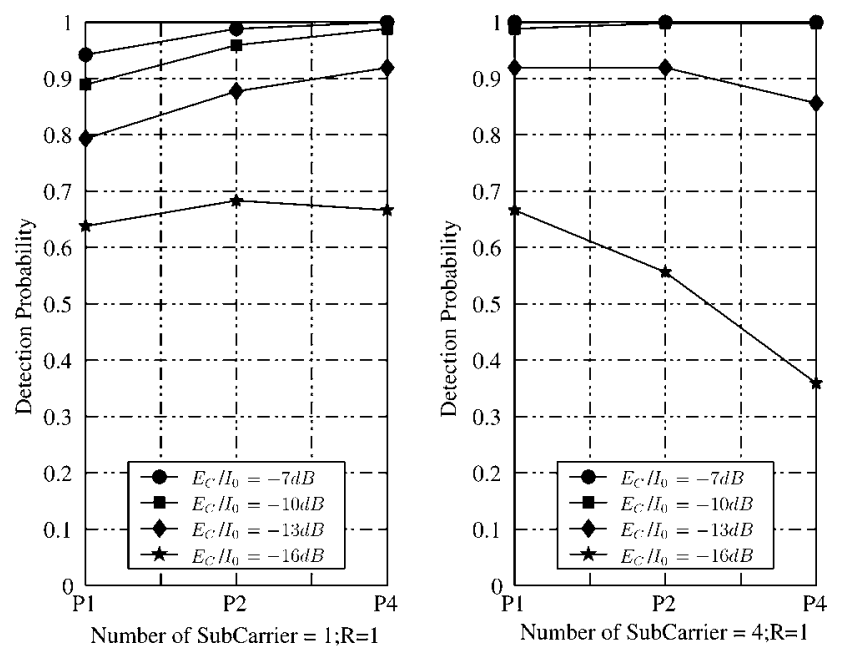

Fig. $7 P_{D}$ of the differentially coherent scheme against the number of transmit antennas for $P=1,2$ as well as 4 , the number of receive antennas for $P=1$ as well as the number of subcarriers for both $U=1$ (left figure) and $U=4$ (right figure) parameterised by the $E_{c} / I_{o}$ 

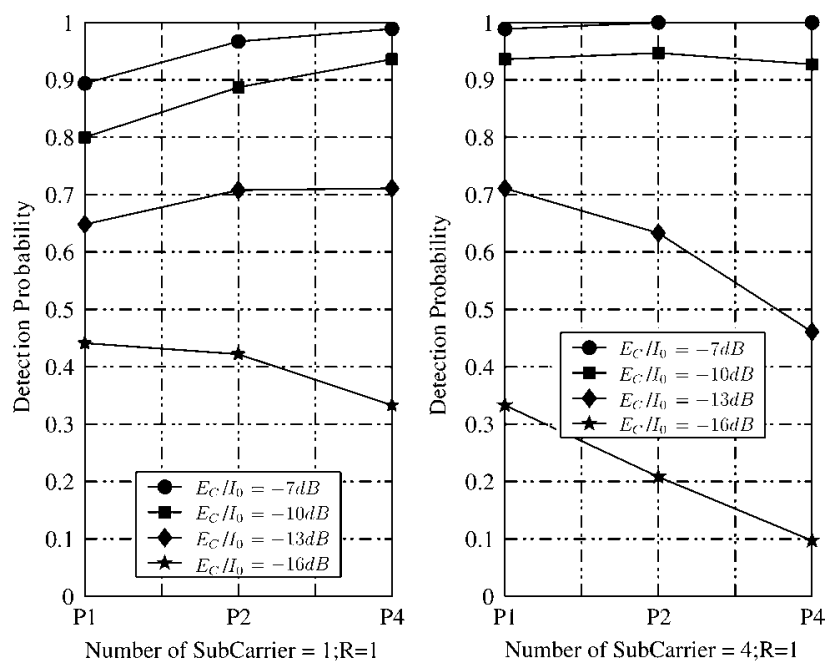

Fig. $8 P_{D}$ of the non-coherent scheme against the number of transmit antennas for $P=1,2$ as well as 4, the number of receive antennas for $P=1$ as well as the number of subcarriers for both $U=1$ (left figure) and $U=4$ (right figure) parameterised by the $E_{c} / I_{o}$

the employment of multiple transmitters and multiple subcarriers.

Both Figs. 7 and 8 document the relationship between $P_{\mathrm{D}}$ and the number of transmit antennas required by both the DDSS-assisted DC and NC code acquisition schemes for approaching their lowest possible MAT. Explicitly, the results of Figs. 7 and 8 were recorded against the number of subcarriers $(U=1$ and 4$)$, parameterised with both the number of transmit antennas and with different values of $E_{\mathrm{c}} / I_{\mathrm{o}}$, respectively. The correct detection probabilities seen in both Figs. 7 and 8 were obtained assuming a false locking probability of $P_{\mathrm{F}}=5 \times 10^{-4}$ for all scenarios considered, since the reliable operational range in the verification mode of the DDSS-assisted code acquisition scheme is a false alarm probability spanning from $10^{-3}$ to $10^{-4}$. The left illustration of Fig. 7 characterises the scenario of $U=1$, whereas the one at the right of Fig. 7 is valid for the arrangement having $U=4$. When increasing the number of transmit antennas, the curves recorded for $E_{\mathrm{c}} / I_{\mathrm{o}}$ values spanning from -7 to $-13 \mathrm{~dB}$ at the left of Fig. 7 indicates a $P_{\mathrm{D}}$ improvement, although at low $E_{\mathrm{c}} / I_{\mathrm{o}}$ values, say at $E_{\mathrm{c}} / I_{\mathrm{o}}=-16 \mathrm{~dB}$, the opposite is true. On the other hand, the results seen at the right of Fig. 7 indicate that $P_{\mathrm{D}}$ tends to decrease, as both the number of transmit antennas and the number of subcarriers increases, when typical $E_{\mathrm{c}} / I_{\mathrm{o}}$ values are encountered. The results of Fig. 8 also exhibit similar performance trends. However, due to the performance loss of the NC scheme in comparison to the DC arrangement, the achievable $P_{\mathrm{D}}$ performance further deteriorates over the $E_{\mathrm{c}} / I_{\mathrm{o}}$ values considered.

The main reasons for the afore-mentioned performance trends are further justified by information theoretic considerations in NC MIMO scenarios [19-22]. Finally, when considering the design of MIMO-aided code acquisition scheme, the following guidelines may be inferred.

1. Using multiple transmit antennas typically leads to an MAT performance degradation, except for the ' $\mathrm{P} 2 R 1$ ' senario. Using a relatively low number of chips, over which integration or accumulation is carried out further limits the benefits of MTMR antennas.

2. Using multiple receive antennas increases the achievable receiver diversity gain and has the potential of compensating for the MAT degradation imposed by the low per-branch power of multiple transmitters.

3. For the sake of acquiring the initial timing information of the received paths, specificially designed preambles, such as that of the primary synchronisation channel (P-SCH) of W-CDMA [23] combined with time-switched transmit diversity (TSTD) might be recommended, which is capable of achieving a diversity gain with the aid of a single transmit antenna $[19,20]$. The pilot channel may also be used for other purposes, such as frequency error correction and channel estimation.

\section{Conclusion}

In this contribution, we characterised the MTMR antenna-assisted-diversity effects on the attainable code acquisition performance of both DC and NC code acquisition schemes in the inter-cell synchronous MC-DS-CDMA downlink. The probabilities of correct detection and false alarm have been formulated analytically and the achievable MAT performance was characterised. Surprisingly, our conclusions suggest that increasing both the number of transmit antennas and that of the subcarriers in an MIMO-assisted MC-DS-CDMA system leads to combining the low-energy signals of both the transmit antennas and the subcarriers, which may increase the MAT by an order of magnitude, in particular, in the critical scenario, when the SINR is relatively low. Consequently, our future research will be aimed at specifically designing turbo-like iterative acquisition schemes [4, 24] for MIMO-aided MC transmission systems.

\section{Acknowledgment}

The authors acknowledge the financial support of the Ministry of Information and Communication (MIC), Republic of Korea and the European Union under the auspices of the Phoenix and Newcom projects and that of the EPSRC.

\section{References}

1 Gesbert, D., Shafi, M., Shiu, D.S., Smith, P.J., and Naguib, A.: 'From theory to practice: an overview of MIMO space-time coded wireless systems', IEEE J. Sel. Areas Commun., 2003, 21, (3), pp. 281-302

2 Yang, L.-L., and Hanzo, L.: 'Multicarrier DS-CDMA: a multiple access scheme for ubiquitous broadband wireless communications', IEEE Commun. Mag., 2003, 41, (10), pp. 116-124

3 Yang, L.-L., and Hanzo, L.: 'Performance of generalized multicarrier DS-CDMA over Nakagami-m fading channels', IEEE Trans. Commun.,' 2002, 50, (6), pp. 956-966

4 Hanzo, L., Yang, L.-L., Kuan, E.-L., and Yen, K.: 'Single- and multi-carrier DS-CDMA' (John Wiley \& Sons, 2003), p. 1077

5 Hanzo, L., Münster, M., Choi, B.J., and Keller, T.: 'OFDM and MC-CDMA for broadcasting multi-user communications, WLANs and broadcasting' (John Wiley \& Sons, 2003), p. 978

6 Lee, B.-G., and Kim, B.-H.: 'Scrambling techniques for CDMA communications' (Kluwer Academic Publishers, 2001), Ch. 2 and 3, pp. 15-37

7 Yang, L.-L., and Hanzo, L.: 'Serial acquisition of DS-CDMA signals in multipath fading mobile channels', IEEE Trans. Veh. Technol., 2001, 50, (2), pp. 617-628

8 Yang, L.-L., and Hanzo, L.: 'Serial acquisition performance of single-carrier and multicarrier DS-CDMA over Nakagami-m fading channels', IEEE Trans. Wirel. Commun., 2002, 1, (4), pp. 92-702

9 Won, S.H., and Kim, Y.J.: 'Performance analysis of multi-path searcher for mobile station in W-CDMA system employing transmit diversity', Electron. Lett., 2003, 39, (1), pp. 137-139

10 Won, S.H., and Hanzo, L.: 'Analysis of serial search based code acquisition in the multiple transmit antenna aided DS-CDMA downlink'. Vehicular Technology Conf., Dallas, USA, September 2005, pp. $98-102$ 
11 Zarrabizadeh, M.H., and Sousa, E.S.: 'A differentially coherent PN code acquisition receiver for CDMA systems', IEEE Trans. Commun., 1997, 45, (11), pp. 1456-1465

12 Lin, J.-C.: 'Differentially coherent PN code acquisition with full-period correlation in chip-synchronous DS/SS receivers', IEEE Trans. Commun., 2002, 50, (5), pp. 698-702

13 Shin, O.S., and Lee, K.B.: 'Differentially coherent combining for double-dwell code acquisition in DS-CDMA systems', IEEE Trans. Commun., 2003, 51, (7), pp. 1046-1050

14 Viterbi, A.J.: 'CDMA: principles of spread spectrum communication' (Addison-Wesley, 1995), Ch. 3, pp. 39-75

15 Park, H.-R., and Kang, B.-J.: 'On serial search code acquisition for direct-sequence spread spectrum system: an applicaion to IS-95 CDMA system'. Vehicular Technology Conf., 25-28 July 1995, vol. 1, pp. $291-295$

16 Proakis, J.G.: 'Digital communications' (McGraw-Hill, 2001, 4th edn.), Ch. 2, pp. 17-79

17 Holm, H., and Alouini, M.-S.: 'Sum and difference of two squared correlated nakagami variates in connection with the McKay distribution', IEEE Trans. Commun., 2004, 52, (8), pp. $1367-1376$
18 Park, H.R.: 'Performance analysis of a double-dwell serial search technique for cellular CDMA networks in the case of multiple pilot signals', IEEE Trans. Veh. Technol., 1995, 48, (6), pp. $1819-1830$

19 Zheng, L., and Tse, D.N.C.: 'Communication on the grassmann manifold: a geometric approach to the noncoherent multiple-antenna channel', IEEE Trans. Inform. Theory, 2002, 48, (2), pp. 359-383

20 Rao, C., and Hassibi, B.: 'Analysis of multiple-antenna wireless links at low SNR', IEEE Trans. Inform. Theory, 2004, 50, (9), pp. 2123-2130

21 Marzetta, T.L., and Hochwald, B.M.: 'Capacity of a mobile multiple-antenna communication link in rayleigh flat fading', IEEE Trans. Inform. Theory, 1999, 45, (1), pp. 139-157

22 Ray, S., Medard, M., and Zheng, L.: 'On MIMO capacity in the ultra-wideband regime, signals, systems and computers'. Conf. Record of the 38th Asilomar Conf., 2004, vol. 2, pp. 1516-1520

23 Wang, Y.-P.E., and Ottosson, T.: 'Cell search in W-CDMA', IEEE J. Sel. Areas Commun., 2000, 18, (8), pp. 1470-1482

24 Yang, L.-L., and Hanzo, L.: 'Acquisition of m-sequences using recursive soft sequential estimation', IEEE Trans. Commun., 2004, 52, (2), pp. 199-204 